\title{
Traumatismo da medula espinhal em cães e gatos: estudo prospectivo de $57 \operatorname{casos}^{1}$
}

\author{
Daniela S. Mendes² e Mônica V. Bahr Arias ${ }^{2 *}$
}

\begin{abstract}
Mendes D.S. \& Bahr Arias M.V. 2012. [Spinal cord injuries in dogs and cats: prospective study of 57 cases.] Traumatismo da medula espinhal cães e gatos: estudo prospectivo de 57 casos. Pesquisa Veterinária Brasileira 32(12):1304-1312. Departamento de Clínicas Veterinárias, Universidade Estadual de Londrina, Rodovia Celso Garcia Cid, PR 445 Km 380, Campus Universitário, Londrina, PR 86051-990, Brazil. E-mail: vicky@uel.br

Spinal cord injury is a common cause of neurological dysfunction in dogs and cats. Lesions in these species are due to various types of accident, which can cause sequelae that impair the patient for life as a pet, or cause life-threatening injury. The main purpose of this work was the accompaniment of animals with spinal cord trauma seen from August 2009 to November 2010 at the Veterinary Hospital of Universidade Estadual de Londrina, studying the epidemiology and etiology, risk factors, spinal cord segments most affected, outcome of conservative or surgical treatment, ratio of time of patient care with recovery, efficacy and side effects of neuroprotective drugs, complications, sequels and evolution of patients. During this period we monitored 57 animals ( 48 dogs and nine cats). We observed a predominance of males $(68 \%)$ and indoors $(79 \%)$. The main cause of injury was being hit by a car (66\%). The time between injury and first attendance was less than eight hours in $42 \%$ of cases and more than a day in $51 \%$. The spinal segment most affected was the thoracolumbar (52\%). Twenty animals were euthanized after the initial attendance due to poor prognosis. The conservative treatment such as rest and/or external immobilization was performed on 29 animals, and a good outcome was seen in $72.4 \%$ of this patients: total functional recovery was observed in 17 (58.6\%) animals and partial functional recovery was seen in four (13.8\%). Four animals did not recover, and four animals died. Surgical treatment was performed in eight patients, and three animals recovered, one patient did not recover and four animals died or were subjected to euthanasia due to complications during intraoperative or postoperative period. Conservative treatment was a viable therapy, mainly in dogs with cervical spinal cord trauma.
\end{abstract}

INDEX TERMS: Nervous system, spinal cord, vertebral fracture, dogs and cats.

RESUMO.- 0 traumatismo da medula espinhal é uma causa comum de disfunção neurológica em cães e gatos. Lesões nestas espécies ocorrem devido a vários tipos de acidentes, podendo ocasionar sequelas que prejudicam o paciente como animais de estimação ou lesões que comprometam a vida. 0 objetivo principal deste estudo foi a monitoração intensiva de animais com lesão medular atendidos entre 8/2009 e 11/2010 no Hospital Veterinário da Universida-

\footnotetext{
${ }^{1}$ Recebido em 22 de setembro de 2011.

Aceito para publicação em 23 de abril de 2012.

${ }^{2}$ Departamento de Clínicas Veterinárias, Universidade Estadual de Londrina (UEL), Campus Universitário, Rodovia Celso Garcia Cid, PR 445 Km 380, Londrina, PR 86051-990, Brasil. *Autor para correspondência: vicky@uel.br
}

de Estadual de Londrina, estudando os fatores epidemiológicos e etiológicos, fatores de risco, segmentos medulares mais afetados, resultados dos tratamentos conservativo ou cirúrgico, relação entre o tempo de atendimento e a recuperação do animal, eficácia e efeitos colaterais do succinato sódico de metilprednisolona, complicações, sequelas e evolução do quadro. Durante este período foram acompanhados 57 animais (48 cães e nove gatos). Observou-se predominância de animais machos (68\%) e domiciliados (79\%). A principal causa de lesão foi o atropelamento (66\%). 0 tempo entre o trauma e o atendimento foi menos de oito horas em $42 \%$ dos casos e mais de um dia em $51 \%$. 0 segmento medular mais atingido foi o toracolombar (52\%). Vinte animais foram submetidos à eutanásia após o atendimento inicial devido ao prognóstico reservado. 0 trata- 
mento conservativo com repouso e/ou imobilização externa foi realizado em 29 animais e um bom resultado com esta modalidade foi obtida em $72,4 \%$ dos casos: houve recuperação funcional total em 17 (58,6\%) animais e parcial em quatro $(13,8 \%)$. Quatro animais não se recuperaram e quatro animais morreram. Oito pacientes foram submetidos ao tratamento cirúrgico, sendo que três animais recuperaram-se, um paciente não apresentou recuperação e quatro morreram ou foram submetidos a eutanásia devido a complicações no trans ou pós-operatório. 0 tratamento conservativo foi viável, principalmente em cães com lesão medular cervical.

TERMOS DE INDEXAÇÃO: Sistema nervoso, medula espinhal, fratura vertebral, cães e gatos.

\section{INTRODUÇÃO}

Lesões traumáticas à coluna vertebral e medula espinhal ocorrem frequentemente na medicina veterinária e humana, levando a sequelas devastadoras, como perda parcial ou completa das funções motoras, sensoriais e viscerais (Bergman 2000). A medula espinhal e raízes associadas à ela na maior parte dos casos são comprometidas após trauma às vértebras, devido à compressão ou contusão do tecido neural, entretanto devido a fatores anatômicos das vértebras, ligamentos, segmento medular, localização dos corpos celulares dos neurônios e diâmetro do canal vertebral, pode ser observado grande deslocamento vertebral com pouca lesão ao tecido nervoso, bem como grande lesão ao tecido nervoso sem grande comprometimento das vértebras (Jeffery 2010). As consequências do trauma medular em veterinária, dependendo do segmento lesionado, podem levar à incapacidade permanente, morte (Arias et al. 2007) ou eutanásia (Bergman 2000). Os impactos externos podem resultar em fratura, subluxação, luxação vertebral (Bagley et al. 1999), hemorragia, hematoma, edema medular, lesões compressivas, laceração medular ou das raízes associadas, incluindo a cauda equina, que resultam em disfunções neurológicas em diferentes graus (Grasmueck \& Steffen 2004). Em 155 cães atropelados, a morte ou razão para eutanásia em 27,7\% dos casos foi o trauma medular (Fighera et al. 2008). O tratamento é um desafio tanto para a medicina humana como para a veterinária, e deve ser considerado emergência, visto que a decisão rápida e adequada aumenta as chances de recuperação funcional (Bergman et al. 2000a, 2000c, Arias et al. 2007).

Hawthorne et al. (1999), Olby et al. (2003) e Grasmueck \& Steffen (2004) realizaram estudos retrospectivos em cães e gatos com trauma espinhal, constatando que o prognóstico é pobre para a recuperação funcional após fratura ou luxação vertebral com perda da percepção da dor profunda, entretanto, alguns animais recuperaram a função motora e a continência urinária. No estudo de Grasmueck \& Steffen (2004) todos os gatos com paraplegia grau 5 foram submetidos a eutanásia, pois a maioria apresentou mielomalácia hemorrágica. Até o momento não existe tratamento capaz de restaurar as funções da medula espinhal lesada (Defino 1999), persistindo como principal conduta a prevenção (Souza Junior 2003, Campos et al. 2008). O SSMP (succinato sódico de metilprednisolona) é um fármaco neuroprotetor que ainda é bastante utilizado na lesão medular aguda em humanos e animais, devido aos seus efeitos benéficos na cascata de eventos secundários que se desenvolvem após o trauma medular (Arias et al. 2007, Kube \& Olby 2008). Porém, várias pesquisas têm mostrado que além de haver poucos benefícios no tecido lesado com o seu uso, ocorrem várias complicações sistêmicas (Bagley et al. 1999, Bergman et al. 2000c, Arias et al. 2007).

Como a frequência de atendimento de cães e gatos com trauma medular na rotina do Hospital Veterinário da Universidade Estadual de Londrina é elevada, o objetivo deste trabalho foi o acompanhamento dos animais com histórico de trauma, estudando os aspectos epidemiológicos e etiológicos, os fatores de risco, os segmentos medulares afetados, os resultados obtidos com os tratamentos conservativo e cirúrgico quando realizados, a relação entre o tempo de atendimento e a recuperação do animal, a eficácia e efeitos colaterais do SSMP, complicações, sequelas e a evolução do quadro.

\section{MATERIAL E MÉTODOS}

Durante o período de 8/2009 a 11/2010 foram acompanhados 57 animais (48 cães e nove gatos) atendidos no Hospital Veterinário da Universidade Estadual de Londrina com lesão na coluna vertebral envolvendo a medula espinhal ou a cauda equina ocasionada por trauma externo. Após o exame clínico, aplicação de analgésicos e estabilização do quadro hemodinâmico, utilizando-se fluidoterapia com ringer lactato ou salina a $0,9 \%$ e oxigenioterapia quando indicado, foram realizados os exames neurológico e radiográfico. Devido ao histórico de trauma associado à presença de deficiências neurológicas indicando lesão medular, principalmente quando era observado um desnível na coluna levando a suspeita de fratura vertebral instável, os animais foram imobilizados em uma superfície rígida, para prevenir lesões adicionais à medula espinhal causadas pela movimentação das vértebras instáveis (Sharp \& Wheeler 2005, Arias et al. 2007, Kube \& Olby 2008, Araújo et al. 2009, Olby 2010). As informações epidemiológicas como raça, sexo, idade, peso, moradia, causa da lesão, tempo entre trauma e atendimento, lesões associadas, alterações do exame neurológico e radiográfico foram anotados no prontuário do paciente. Os animais foram acompanhados por reavaliações periódicas e contato telefônico com o proprietário.

O diagnóstico de lesão medular foi realizado com base na anamnese, resultado dos exames físico, neurológico e radiográfico e achados intra-operatórios ou de necropsia. Os locais de lesão medular foram classificados de acordo com o segmento medular afetado e o grau da lesão medular. As lesões foram localizadas em uma das quatro áreas da medula espinhal: cervical (C), cérvico-torácica, toracolombar (TL) e lombossacra, entretanto como os sinais clínicos observados nas doenças na região lombossacra diferem dos sinais observados em outras regiões da medula, devido à presença da cauda equina, optou-se pela separação do segmento lombossacro em lombar (L) e lombossacro (LS), para melhor avaliação dos resultados (Sharp \& Wheeeler 2005, Bruce et al. 2008, De Lahunta \& Glass 2009). Assim, os seguintes graus de lesão medular foram considerados: nos segmentos medulares cervical (C1-C5) e cérvico-torácico (C6-T2) - grau 1 (dor), grau 2 (dor e ataxia), grau 3 (dor, tetraparesia, tetraplegia); segmento toracolombar (T3-L3) e lombar (L4-L6) - grau 1 (dor), grau 2 (ataxia e diminuição da propriocepção), grau 3 (paraplegia), grau 4 (paraplegia e retenção ou incontinência urinária) e grau 5 (idem 4 
associado a perda da sensibilidade dolorosa profunda); segmento lombossacro (L7-S3) - grau 1 (dor), grau 2 (dor e paresia), grau 3 (dor, incontinência urinária, cauda flácida, esfíncteres relaxados) e grau 4 (dor, cauda flácida, retenção urinária).

O diagnóstico da presença ou ausência da sensibilidade dolorosa profunda nos pacientes paraplégicos foi realizado aplicando-se a pressão de uma pinça hemostática no periósteo dos dígitos e observando se o animal manifestava uma resposta consciente, como vocalização ou movimentação da cabeça em direção ao estímulo doloroso, indicando que foi transmitido pela medula espinhal ao encéfalo (Grasmueck \& Steffen 2004, Sharp \& Wheeeler 2005, Arias et al. 2007, Araújo et al. 2009). Considerou-se recuperação funcional total o retorno das funções motora, sensorial e visceral e alívio da dor. Considerou-se recuperação funcional parcial o retorno incompleto destas funções, ou seja, o animal apresentava ataxia ou incontinência urinária ou fecal, mas caminhava. Quando o quadro permaneceu inalterado considerou-se que o animal não apresentou recuperação. No caso do animal apresentar caminhar espinhal, ou seja, capacidade de sustentar o peso com os membros pélvicos (Grasmueck \& Steffen 2004, Araújo et al. 2009), realizar movimentos de locomoção involuntários, mas não apresentar sensibilidade dolorosa profunda, considerou-se como não recuperação.

Foi realizada radiografia simples em todos os animais e, em três pacientes realizou-se também mielografia. Considerou-se subluxação vertebral deslocamento de no máximo $25 \%$ de uma vértebra em relação a outra. Para avaliação radiográfica da instabilidade da fratura, utilizou-se a teoria dos três compartimentos e a fratura foi considerada instável quando dois ou mais compartimentos estivessem comprometidos (Bagley et al. 1999, Sharp \& Wheeler 2005, Araújo et al. 2009, Jeffery 2010). Os critérios para a escolha de tratamento conservativo ou cirúrgico foram a suspeita de instabilidade vertebral ou compressão medular na avaliação radiográfica e o prognóstico obtido após a avaliação do grau de lesão medular pelo exame neurológico (Bagley et al. 1999, Sharp \& Wheeler 2005, Kube \& Olby 2008, Platt \& Olby 2010). Influenciaram ainda na decisão do tratamento a presença de outras lesões associadas ao trauma (Olby 2010), se o animal era domiciliado e a decisão do proprietário.

No caso de opção pelo tratamento conservativo, a imobilização externa foi realizada com aplicação de atadura elástica, algodão ortopédico e tala de alumínio, associado a repouso em canil/ gatil. Os pacientes eram examinados periodicamente para reavaliação da imobilização e de suas complicações e troca da mesma quando estava úmida ou deslocada.

0 resultado final levou em consideração a sobrevivência e recuperação dos pacientes. Os dados foram analisados com o auxílio do programa Epi 6,04 - CDC Atlanta através do teste de Qui-quadrado ou Exato de Fisher, com nível de significância de $5 \%$, avaliando-se a sobrevivência em relação à idade, tempo entre trauma e atendimento, segmento medular e presença de lesões associadas. Avaliou-se também a recuperação dos pacientes em relação à presença de dor profunda e a administração de succinato sódico de metilprednisolona (SSMP).

\section{RESULTADOS E DISCUSSÃO}

Dos 57 animais atendidos, 29 pacientes sobreviveram $(50,8 \%)$, sendo que 25 foram submetidos ao tratamento conservativo e quatro ao tratamento cirúrgico. (Quadros 1 e 2). Entre os 28 animais não sobreviventes (49,2\%), 20 foram submetidos à eutanásia, quatro foram submetidos a tratamento conservativo e quatro a tratamento cirúrgico. Ao avaliar-se o número de sobreviventes em relação às
Quadro 1. Perfil epidemiológico, características clínicas e evolução em 48 cães e nove gatos após trauma vertebromedular ou em raízes nervosas atendidos no HV da UEL

(Londrina, 2011)

\begin{tabular}{|c|c|c|c|c|c|}
\hline & \multicolumn{2}{|c|}{ Cães } & \multicolumn{2}{|c|}{ Gatos } \\
\hline & & \multicolumn{4}{|c|}{ Sobrevivência } \\
\hline & & Sim & Não & Sim & Não \\
\hline \multirow[t]{2}{*}{ Sexo } & Macho: & 17 & 19 & 2 & 1 \\
\hline & Fêmea: & 7 & 5 & 3 & 3 \\
\hline \multirow[t]{4}{*}{ Idade } & $<1$ ano: & 4 & 3 & 2 & 1 \\
\hline & 1 a 5 anos: & 14 & 10 & 2 & 3 \\
\hline & 5 a 10 anos: & 3 & 6 & 1 & 0 \\
\hline & > 10 anos: & 3 & 5 & 0 & 0 \\
\hline \multirow[t]{3}{*}{ Peso } & < $5 \mathrm{~kg}:$ & 4 & 4 & 0 & 1 \\
\hline & 5 a $20 \mathrm{~kg}:$ & 20 & 18 & 4 & 2 \\
\hline & > $20 \mathrm{~kg}:$ & 0 & 2 & 1 & 1 \\
\hline \multirow[t]{3}{*}{ Moradia } & Domiciliado: & 18 & 19 & 5 & 3 \\
\hline & Semi-domiciliado: & 0 & 1 & 0 & 1 \\
\hline & Não domiciliado: & 6 & 4 & 0 & 0 \\
\hline \multirow[t]{5}{*}{ Causa } & Atropelamento: & 19 & 17 & 1 & 1 \\
\hline & Agressão por animal: & 3 & 1 & 1 & 0 \\
\hline & Queda: & 0 & 2 & 2 & 0 \\
\hline & Lesão por objeto: & 0 & 0 & 0 & 1 \\
\hline & Desconhecida. & 2 & 4 & 1 & 2 \\
\hline \multirow{3}{*}{$\begin{array}{l}\text { Tempo entre trauma } \\
\text { e atendimento }\end{array}$} & 0 a 8 horas: & 8 & 11 & 3 & 2 \\
\hline & 8 a 24 horas: & 1 & 2 & 1 & 0 \\
\hline & Mais de 24 horas: & 15 & 11 & 1 & 2 \\
\hline \multirow{4}{*}{$\begin{array}{l}\text { Sensibilidade profun } \\
\text { da }(n=40)^{\mathrm{a}} \\
\text { Lesões associadas }\end{array}$} & Com: & 11 & 6 & 1 & 0 \\
\hline & Sem: & 3 & 14 & 1 & 4 \\
\hline & Com: & 7 & 13 & 5 & 2 \\
\hline & Sem: & 17 & 11 & 0 & 2 \\
\hline \multirow{4}{*}{$\begin{array}{l}\text { Segmento medular } \\
\text { afetado }\end{array}$} & Cervical: & 4 & 0 & 2 & 0 \\
\hline & Toracolombar: & 12 & 13 & 1 & 4 \\
\hline & Lombar: & 2 & 7 & 1 & 0 \\
\hline & Lombossacro & 6 & 4 & 1 & 0 \\
\hline \multirow{6}{*}{$\begin{array}{l}\text { Alterações radiográ- } \\
\text { ficas }\end{array}$} & Fratura: & 8 & 6 & 1 & 1 \\
\hline & Luxação: & 4 & 5 & 1 & 0 \\
\hline & Fratura/Luxação: & 3 & 3 & 1 & 1 \\
\hline & Fratura por compressão: & 1 & 5 & 1 & 0 \\
\hline & Subluxação: & 3 & 1 & 1 & 2 \\
\hline & Sem alterações: & 5 & 4 & 0 & 0 \\
\hline \multirow[t]{3}{*}{ Conduta } & Tratamento Conservativo: & 20 & 4 & 5 & 0 \\
\hline & Tratamento Cirúrgico: & 4 & 3 & 0 & 1 \\
\hline & Eutanásia: & 0 & 17 & 0 & 3 \\
\hline
\end{tabular}

aSomente animais com lesão no segmento Toracolombar ou Lombar, pois animais com lesão no segmento Cervical ou Lombossacro dificilmente perdem a sensibilidade profunda

Quadro 2. Recuperação funcional em 24 cães e cinco gatos sobreviventes após trauma medular atendidos no HV da UEL (Londrina, 2010)

\begin{tabular}{llccc}
\hline \multirow{2}{*}{ Sensibilidade profunda } & & \multicolumn{3}{c}{ Recuperação funcional } \\
\cline { 3 - 5 } & & Total & Parcial & Sem \\
\hline \multirow{2}{*}{ Lesões associadas } & Sem: & 7 & 4 & 1 \\
\multirow{2}{*}{ Tratamento realizado } & Com: & 0 & 0 & 4 \\
& Sem: & 10 & 0 & 2 \\
Tempo entre trauma e & Conservativo: & 17 & 5 & 3 \\
atendimento & Cirúrgico: & 2 & 1 & 4 \\
& 0 a 8 horas: & 10 & 0 & 2 \\
& 8 a 24 horas: & 1 & 0 & 1 \\
& Após 24 hrs: & 8 & 5 & 2
\end{tabular}

a Somente animais com lesão no segmento Toracolombar ou Lombar; pois animais com lesão no segmento Cervical ou Lombossacro dificilmente perdem a sensibilidade profunda. 
variáveis: idade $(\mathrm{P}=0,344)$, tempo entre trauma e atendimento $(\mathrm{P}=0,692)$, segmento medular afetado $(\mathrm{P}=0,037)$ e lesões associadas $(\mathrm{P}=0,511)$, somente o local da lesão medular apresentou influência sobre a mortalidade. Dos 30 animais com lesão toracolombar e 10 com lesão lombar, $57 \%(n=17)$ e $70 \%(n=7)$ morreram ou foram submetidos à eutanásia respectivamente, e $100 \%$ dos animais $(n=6)$ com lesão cervical sobreviveram (Quadro 1).

Vinte pacientes foram submetidos à eutanásia após o atendimento inicial: 17/20 foram eutanásias indicadas devido ao prognóstico ruim, obtido após o exame neurológico e constatação da perda da sensibilidade dolorosa profunda e observação de luxação/fratura com deslocamento no exame radiográfico, e 3/20 eutanásias não indicadas, sendo a decisão do proprietário (Fig.1). À necropsia realizada em seis destes pacientes, constatou-se interrupção anatômica da medula espinhal em três, mielomalacia focal e hemorragia em dois, e hemorragia difusa no espaço subdural e tecido nervoso em um. As lesões graves observadas na medula espinhal destes pacientes corroboraram as informações obtidas no exame neurológico inicial. Embora recuperação funcional já tenha sido descrita em cães com mielomalacia focal, este achado é um indicador de prognóstico ruim, principalmente se associado a perda da dor profunda em casos de trauma medular exógeno, embora o inverso não seja verdadeiro, ou seja, a perda da dor profunda não indica mielomalacia (Grasmueck \& Steffen 2004).

Avaliando-se a presença de dor profunda $(\mathrm{P}=0,001)$ e administração de SSMP $(\mathrm{P}=0,596)$, somente a presença da sensibilidade dolorosa profunda apresentou influência sobre a taxa de recuperação: entre os 40 animais com lesão medular toracolombar e lombar, constatou-se que de 18 animais com a sensibilidade dolorosa profunda preservada, 12 sobreviveram (66,7\%), enquanto que dos 22 animais sem sensibilidade, 18 não sobreviveram ou foram submetidos à eutanásia $(81,8 \%)$. A preservação da sensibilidade dolorosa profunda é o aspecto mais importante para o prognóstico em animais com lesão medular toracolombar (Jeffery \& Blakemore 1999, Bagley et al. 1999, Bergman et al. 2000a, Sharp \& Wheeler 2005, Arias et al. 2007, Platt \& Olby 2010, Webb et al. 2010). A dor profunda é carreada por axônios de diâmetro pequeno que estão localizados mais profundamente na medula espinhal, e é neces-

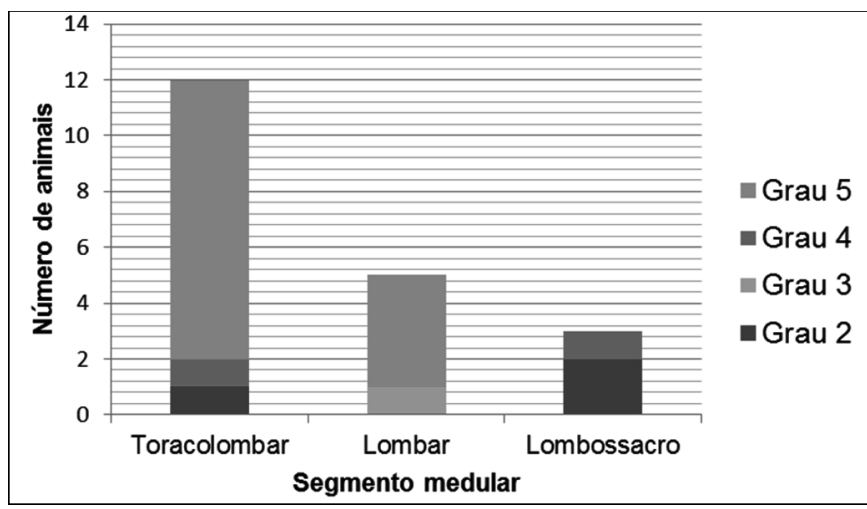

Fig.1. Segmento medular acometido e grau de lesão medular em 20 animais submetidos à eutanásia após o atendimento inicial (Londrina, 2011). sária uma lesão severa na medula espinhal para afetá-los (Bergman et al. 2000a, Olby et al. 2003, Jorge 2009), sendo o prognóstico ruim em animais que perderam a percepção da dor profunda, principalmente quando decorrente de traumas com fratura/luxação vertebral (Bergman et al. 2000a, 2000b, Kube \& Olby 2008, Jorge 2009). Já cães com trauma medular de origem endógena, como a doença do disco intervertebral, com perda da sensibilidade dolorosa profunda, apresentam maior chance de recuperação (Olby et al. 2003, Arias et al. 2007, Kube \& Olby 2008, Webb, Ngan \& Fowler 2010, Santos et al. 2011). Segundo Olby et al.(2003), cães com lesão medular toracolombar grau 5 decorrente de trauma não recuperam a dor profunda, embora possam voltar a caminhar, apresentando como sequela incontinência fecal e urinária. A perda da sensibilidade dolorosa profunda nestes casos está frequentemente associada à transecção medular ou mielomalacia (Araújo et al. 2009). Já animais com paraplegia graus 3 e 4, ou seja, com a sensibilidade dolorosa profunda intacta, apresentam maior chance de recuperação, como visto no presente trabalho, em que nove animais apresentaram recuperação e cinco não (Fig.2). A sensibilidade dolorosa preservada indica que há axônios intactos e de acordo com Webb et al. (2010), são necessários apenas $10 \%$ dos axônios viáveis para desenvolver a capacidade de locomoção.

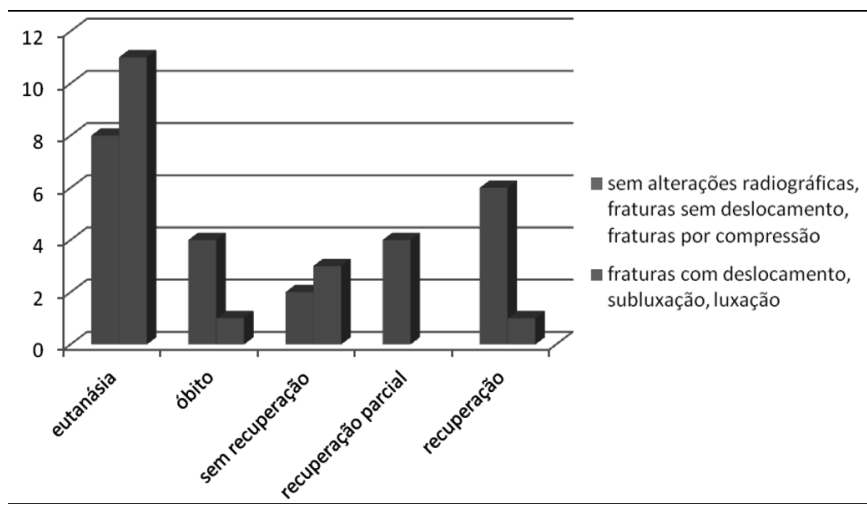

Fig.2. Realização de eutanásia, morte ou evolução do quadro em 40 animais com trauma em vértebras torácicas e lombares, de acordo com as alterações radiográficas observadas.

A análise de imagens radiográficas só permite avaliar o grau de lesão da coluna vertebral, e não da medula espinhal, sendo o exame neurológico soberano para esta avaliação (Araújo et al. 2009), entretanto animais com lesões toracolombares e deslocamento de 30 a $100 \%$ do canal vertebral associado à perda da dor profunda no exame neurológico não terão possibilidade de retorno funcional devido a transecção total da medula espinhal (Araújo et al. 2009, Bali et al. 2009). Isto está de acordo com os resultados do presente trabalho, pois constatou-se que animais apresentando luxações e fraturas com deslocamento maior que 30\% apresentaram menor recuperação quando comparado com animais sem alterações radiográficas, fraturas sem deslocamento ou fraturas compressivas do corpo das vértebras (Fig.2). Entretanto, também pode ser observado que no grupo de animais sem sinais radiográficos, ou fraturas sem deslocamento, houve um número elevado de mor- 
tes/eutanásia devido à gravidade dos sinais clínicos, pois neste grupo muitos animais apresentavam síndrome toracolombar grau 5 (Fig.2). Nos animais com lesão TL ou lombar grau 5 que foram submetidos à necropsia, constatou-se mielomalacia ou secção da medula espinhal. Infelizmente, lesões toracolombares são mais frequentes que em outros segmentos (Sharp \& Wheeler 2005, Bruce et al. 2008, Bali et al. 2009), como constatado neste trabalho.

Entre os 12 animais com lesão toracolombar ou lombar e sensibilidade profunda intacta, 11 pacientes (96\%) recuperaram-se (sete totalmente e quatro parcialmente) e um animal (4\%) não se recuperou, (Quadro 2, Fig.3), porcentagem próxima do descrito por Grasmueck \& Steffen (2004), que observaram $83 \%$ de recuperação nas lesões TL ou LS cranial grau 2 a 4 . Estes mesmos autores, ao realizarem o tratamento cirúrgico em animais com o mesmo tipo de lesão, observaram recuperação em $67 \%$ dos pacientes. No presente estudo, somente dois animais com estes graus de lesão foram operados, entretanto devido à ocorrência de morte em um animal por parada cardiorrespiratória e eutanásia do outro animal devido à sepse, não foi possível comparar os resultados com a literatura consultada.

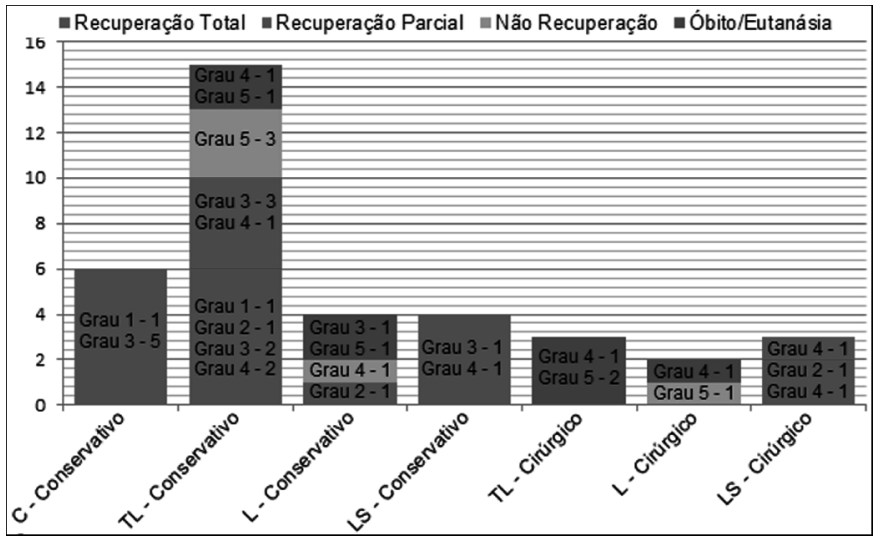

Fig.3. Segmento medular acometido, grau da lesão medular e recuperação funcional em 29 animais submetidos a tratamento conservativo e oito animais submetidos a tratamento cirúrgico após traumatismo espinomedular (Londrina, 2011).

Seis pacientes com lesão medular cervical submetidos a tratamento conservativo apresentaram recuperação total, sendo um paciente com dor cervical, quatro pacientes com tetraparesia e um com tetraplegia (Fig.3). Destes seis animais, quatro pacientes apresentavam subluxação e dois fraturas sem deslocamento. Hawthorne et al. (1999) observaram em animais com lesão cervical submetidos ao tratamento conservativo $89 \%$ de recuperação, concluindo que em animais com lesões cervicais há chance de recuperação com o repouso e imobilização externa. 0 canal vertebral da região cervical tem maior diâmetro quando comparado ao espaço ocupado pela medula espinhal. Já o diâmetro do canal vertebral da coluna torácica e lombar é pequeno. Assim, lesões na coluna cervical causam deficiências mais leves quando comparados a lesões TL, apresentando melhor evolução, como constatado no presente trabalho, mesmo nos casos de luxações da região cervical com instabilidade e deslocamento (Bergman et al. 2000a, Sharp \& Wheeler
2005). Segundo Hawthorne et al. (1999), em cães com lesão medular cervical submetidos à cirurgia a porcentagem de mortes foi de $36 \%$ enquanto que somente $11 \%$ dos cães com lesão cervical submetidos à tratamento conservativo morreram ou foram submetidos à eutanásia. Entretanto os critérios para nortear a escolha entre os dois tratamentos ainda não são totalmente estabelecidos e no presente trabalho o número de animais tratados foi pequeno para corroborar os dados da literatura. Muitas variáveis e particularidades estão envolvidas na escolha do manejo e da melhor conduta no trauma espinomedular, o que dificulta a comparação dos resultados com os vários trabalhos consultados.

Entre os 11 animais com lesão lombossacra, seis pacientes apresentavam dor, cauda flácida e retenção urinária, um paciente apresentou cauda flácida, esfíncteres relaxados e incontinência, e quatro pacientes dor e paresia. $\mathrm{Na}$ radiografia destes animais, observou-se fratura sem deslocamento em seis animais, com deslocamento em um, luxação maior que $50 \%$ em um e avulsão sacrococcígea em três. Observou-se número elevado de eutanásia e morte no grupo de fraturas sem deslocamento, devido às complicações relacionadas ao controle da micção, apesar dos animais voltarem a caminhar. Esta região da coluna vertebral não contém a medula espinhal, e sim a cauda equina, que suporta melhor as deformações causadas por grandes deslocamentos vertebrais e apresenta melhor recuperação após lesões concussivas do que o parênquima medular, assim o grau de deslocamento das vértebras não deve ser usado como parâmetro para o prognóstico para locomoção (Sharp \& Wheeler 2005, Weh \& Kraus 2007). Entretanto, as complicações referentes ao controle da micção e defecação foram os fatores que colaboraram para a decisão de eutanásia, e segundo Sharp \& Wheeler (2005) no exame destes animais, se após um mês, a ausência de movimentação da cauda e da sensibilidade perineal permanecer, o prognóstico para o retorno destas funções é reservado.

Devido ao prognóstico reservado e à necessidade de cuidados específicos, constatou-se a opção pela eutanásia por $74 \%$ dos proprietários dos animais com perda da sensibilidade dolorosa profunda. Observa-se semelhança entre os resultados apresentados neste estudo e aqueles obtidos por Olby et al. (2003), Grasmueck \& Steffen (2004) e Bruce et al. (2008), onde a maioria dos animais com lesões severas que resultaram na perda da sensibilidade profunda foram submetidos a eutanásia após o exame inicial devido ao prognóstico desfavorável. No presente estudo, entre os quatro animais sobreviventes que perderam a sensibilidade profunda nenhum apresentou recuperação, sendo o acompanhamento destes animais realizado durante no mínimo três meses após o atendimento, confirmando-se a influência negativa da perda da dor profunda sobre a recuperação dos pacientes e na escolha da eutanásia devido ao prognóstico reservado.

Também influenciou na decisão da eutanásia se o animal era domiciliado ou não e a presença de lesões que encareceriam o tratamento, devido à necessidade de cirurgia ou maior tempo de internamento: entre os 20 animais submetidos à eutanásia (17 cães e três gatos), cinco (25\%) 
eram não domiciliados, um era semi-domiciliado (5\%), e oito $(40 \%)$ domiciliados tinham acesso à rua, sendo que 11 animais (55\%) apresentaram lesões concomitantes em outros sistemas. Segundo estudo realizado por Fighera et al. (2008b) o trauma foi um dos principais fatores para a ocorrência de morte ou eutanásia em cães adultos no Rio Grande do Sul, assim como no estudo de Bentubo et al. (2008), que observaram que o trauma foi a terceira causa mais comum de morte de cães em São Paulo. Bentubo et al. (2008) recomendaram ainda que os médicos veterinários orientem os proprietários de animais quanto à adoção de medidas de segurança que minimizem tais riscos, como o uso de guias e coleiras durante os passeios.

Os segmentos medulares mais acometidos foram o toracolombar/lombar $(68,1 \%)$ e o lombossacro $(19,3 \%)$ (Quadro 1), o que está de acordo com o estudo de Zotti et al. (2011), que realizaram um estudo do momento de resistência das vértebras e da coluna vertebral com um scanner radiográfico e compararam os resultados com a frequência de fraturas nos diversos segmentos vertebrais, confirmando que esta parte da coluna é o segmento mais fraco do esqueleto axial e que sofre a carga mais alta de forças estáticas e dinâmicas. Segundo os mesmos autores, outros fatores como a posição do animal no momento do trauma e tipo de forças aplicadas também influenciam na evolução do quadro. A localização da lesão medular afeta ainda a gravidade dos sinais clínicos (Bergman et. al. 2000a, Jorge 2009). Segundo Fighera et al. (2008a), é 19 e 13 vezes mais frequente um cão vítima de atropelamento morrer ou sofrer eutanásia em decorrência de traumatismo espinhal-medular ocasionado por fratura das vértebras torácica ou lombares, respectivamente, do que por fraturas de vértebras cervicais. No estudo de Hawthorne et al. (1999), assim como neste, nenhum animal com lesão cervical apresentou perda da sensibilidade profunda, provavelmente pelo maior diâmetro do canal nesta região e tipo de forças atuantes, caso contrário poderia ocorrer paralisia dos músculos respiratórios e morte (Sharp \& Wheeler 2005, Platt \& Olby 2010).

Observou-se predominância de animais machos (68\%), domiciliados (79\%), com menos de 5 anos de idade (68\%), peso médio 9,8 kg em cães e 3,3 kg em gatos. As principais causas de trauma foram atropelamentos (66\%), agressão por outro animal (9\%) e quedas (7\%). Olby et al. (2003) realizaram um estudo em cães com lesão medular no segmento TL após trauma externo, e assim como neste estudo, observaram maior frequência em cães machos e jovens, provavelmente devido ao comportamento imprudente destes animais (Chai, Johnston \& Shamir, 2008). Entretanto, Grasmueck \& Steffen (2004), em estudo com gatos com lesão medular torácica ou lombar após trauma externo, observaram predominância de fêmeas, sendo as causas principais de lesão medular o atropelamento, seguido por queda. No presente trabalho o atropelamento também foi a causa mais comum, mas como citado por Chai et al. (2008) e constatado por Fighera et al. (2008b), a segunda causa mais comum de lesão medular foi a agressão por outros animais, cuja prevalência relatada está entre 3,8\% e 14,3\% dos casos (Chai et al. 2008).
0 atendimento foi realizado em até 8 horas após o trauma em $42 \%$ dos casos e após um dia em $51 \%$, sendo que o tempo mínimo foi de 25 minutos e o máximo de 28 dias (Quadro 1). Apesar do tempo entre trauma e atendimento não ter influenciado na recuperação do paciente, mais da metade dos animais foi trazido mais de um dia após a ocorrência da lesão. Os proprietários devem ser orientados a trazer os animais precocemente, para proceder-se à analgesia, avaliação e normalização da volemia, imobilização do paciente para evitar lesões mecânicas adicionais à medula espinhal e tratamento das lesões sistêmicas associadas. Uma intervenção rápida pode limitar a extensão da lesão neuronal, favorecendo a recuperação do paciente (Bergman et al. 2000a, 2000c, Sharp \& Wheeler 2005, Arias et al. 2007, Platt \& Olby 2010). A coluna vertebral instável, vértebras deslocadas e a presença de hematoma, ou seja, as lesões primárias levam a maior compressão medular, o que afeta a perfusão do tecido nervoso e inicia uma série de eventos secundários causando inflamação, liberação de radicais livres e maior destruição tecidual (Olby 2010).

Vinte e nove animais (24 cães e cinco gatos) foram submetidos ao tratamento conservativo (Quadro 1, Fig.3), que consistiu em analgesia, confinamento e repouso. Em 10 pacientes foi associada imobilização externa. Obteve-se bom resultado em $72,4 \%$ dos casos: houve recuperação funcional total em 17 (58,6\%) animais e parcial em quatro $(13,8 \%)$. Quatro animais (14\%) não apresentaram melhora e quatro animais (14\%) morreram (Fig.2). A causa da morte não foi diagnosticada em três animais e um animal apresentava fraturas concomitantes em pelve e membro, ocorrendo quadro de sepse após cinco dias de internamento. Oito animais foram submetidos ao tratamento cirúrgico (estabilização e/ou descompressão), em vértebras torácicas (2), toracolombares (1), lombares (2) e lombossacras (3). No tratamento da lesão medular existem as opções de tratamento conservativo ou cirúrgico. 0 tratamento cirúrgico é necessário quando a fratura é instável ou em casos de compressão traumática da medula espinhal pela vértebra instável, por extrusão traumática do disco ou hematoma (Bagley el al. 1999, Jeffery 2010), ou em caso de piora do quadro após tratamento conservativo (Chai, Johnston \& Shamir, 2008), sendo os objetivos da cirurgia a estabilização da coluna vertebral e a descompressão medular, prevenindo assim lesões adicionais à medula espinhal, causadas pelas vértebras instáveis quando estas se deslocam quando o animal se move (Bergman et al. 2000b, Jeffery 2010). Em alguns casos selecionados, onde haja mínima instabilidade vertebral ou poucas alterações neurológicas, o tratamento conservativo pode ser tão benéfico quanto o tratamento cirúrgico, e consiste em confinamento por quatro a seis semanas, analgesia, controle da micção e uso de imobilização externa no caso de fraturas e subluxações (Bagley et al. 1999, Bergman et al. 2000b).

A instabilidade vertebral no contexto de fraturas e luxações vertebrais é difícil de definir em alguns casos, mesmo aplicando-se a teoria dos três compartimentos, pois a posição da vértebra em uma imagem plana não necessariamente reflete a amplitude dos movimentos que podem ocorrer no segmento afetado, nem a extensão dos movimentos que 
ocorreu no momento do trauma (Jeffery 2010). Em alguns pacientes, devido à fatores que contra-indicaram a realização de cirurgia imediata, a imobilização externa foi aplicada, e devido à melhora do quadro neurológico, optou-se pela não realização do procedimento cirúrgico. Segundo Jeffery (2010), a estabilização cirúrgica apresenta inúmeras vantagens sobre o tratamento conservador, mas existem autores que demonstraram que a imobilização externa também pode ser benéfica mesmo em casos mais graves (Hawthorne et al. 1999, Grasmueck \& Steffen 2004, Jeffery 2010). Deve ser lembrado que existem desvantagens com o uso de imobilização externa, como piora do quadro neurológico, assaduras e úlceras de pressão, intolerância à tala, aumento da temperatura do animal e dificuldade no manejo da micção e defecação (Sharp \& Wheeler 2005, Jeffery 2010). No presente estudo dois pacientes retiraram a tala antes do tempo recomendado, e dois apresentaram assaduras na região abdominal, não invibializando o resultado final.

Entre os oito animais submetidos a tratamento cirúrgico, três caninos apresentaram fratura na vértebra L7, sendo que dois foram submetidos a estabilização com parafuso, pinos e polimetilmetacrilato, e um à descompressão e estabilização com a mesma técnica. Os três pacientes voltaram a caminhar, porém um permaneceu com incontinência urinária e fecal. Um cão que apresentou extrusão traumática do disco intervertebral entre vértebras T13-L1 e fratura de fêmur, foi submetido à hemilaminectomia, mas morreu no transoperatório devido a trauma torácico associado. Um canino com luxação lombar foi submetido à eutanásia por apresentar sepse decorrente de infecção urinária, por ter permanecido com sonda uretral de alívio. Segundo Jeffery (2010), tanto o esvaziamento vesical manual incompleto, como a cateterização vesical apresentam risco para o desenvolvimento de infecção como observado neste paciente.

Um cão foi submetido à eutanásia no transoperatório devido à observação de mielomalácia difusa. Dois animais (um canino e um felino) que haviam perdido a sensibilidade profunda, não apresentaram recuperação funcional após a cirurgia, sendo que o felino, após desenvolver caminhar espinhal, morreu devido à ferida e mí́ase nos membros pélvicos três meses depois do trauma e o outro animal usa carrinho de rodas. 0 caminhar espinhal é facilmente desenvolvido em gatos com lesão toracolombar com treinamento e fisioterapia (Grasmueck \& Steffen 2004), mas o fato destes pacientes não apresentarem sensibilidade pode levar a complicações conforme observado no presente estudo.

Lesões sistêmicas concomitantes foram constatadas em 27 animais $(47,4 \%)$, sendo que as lesões mais comuns foram o trauma torácico $(n=8)$, feridas abertas $(n=8)$, fratura de pelve $(n=7)$ e fratura de membros $(n=6)$. Em $53 \%$ dos pacientes (30) houve necessidade de fluidoterapia para normalização da perfusão tecidual. Dos 29 animais submetidos ao tratamento conservativo, 14 (48\%) apresentaram lesões associadas, enquanto que dos oito animais submetidos ao tratamento cirúrgico dois (25\%) apresentaram lesões associadas (Quadro 2). Assim como em pacientes com fraturas de ossos longos que apresentam 38,9\% de probabilidade de trauma torácico associado (Spackman et al. 1984), o trauma medular também pode vir acompanhado deste tipo de lesão e em outros sistemas orgânicos, o que pode ser fatal (Jeffery 2010), pois de modo geral várias estruturas do corpo são afetadas, podendo prejudicar principalmente a circulação e oxigenação, sendo necessária uma avaliação rápida do paciente com ênfase na oxigenação e reposição volêmica para garantir a perfusão medular (Fontelles \& Mantovani 2000, Jeffery, 2010), o que foi realizado no presente trabalho no atendimento inicial. Trunkey (1983) descreveu três picos de mortalidade pós-traumática em humanos: o primeiro ocorre logo após o trauma e deve-se a lesões cerebrais, medula espinhal alta, coração ou grandes vasos. 0 segundo pico ocorre até uma hora após o trauma, e nesta fase é necessário diagnosticar e tratar situações que põem em risco a vida do paciente, como pneumotórax, lesões a órgãos abdominais ou hematoma subdural encefálico. 0 terceiro pico ocorre dias ou semanas após o trauma e deve-se a sepse e a falência múltipla de órgãos. Hawthorne et al. (1999) observaram em cães índices de lesões concomitantes semelhantes ao constatado neste trabalho, e muitas destas lesões e complicações podem ter colaborado para a ocorrência de morte de vários pacientes.

Entre os 29 animais tratados conservativamente, houve recuperação funcional total em 17 animais (58\%), o que representa $29,8 \%$ do total de animais atendidos. Um cão deste grupo de animais apresentou avulsão sacrococcígea apresentando como sequela retenção urinária não responsiva a tratamento médico, e após cirurgia de desnervação dos esfíncteres da bexiga, recuperou-se totalmente. Pacientes com paraplegia que perdem a dor profunda ou apresentam retenção ou incontinência urinária são animais que requerem cuidados intensivos por parte de seus proprietários e as sequelas decorrentes da retenção urinária e presença de feridas e ulcerações podem ser tão graves que podem até levar à morte (Jeffery 2010, Webb et al. 2010). 0 manejo de pacientes paraplégicos ou tetraplégicos inclui a prevenção e tratamento das úlceras de decúbito e da disfunção respiratória, manejo da micção e defecação, prevenção de autotraumatismo e realização de fisioterapia (Bagley et al. 1999, Araújo et al. 2009). No paciente canino com avulsão sacrococcígea que caminhava, mas não urinava, a realização da denervação dos esfíncteres, relatada por Tudury et al. (2006) em gatos com lesão medular toracolombar alta, foi uma opção eficaz, evitando complicações relacionadas à retenção urinária. Observou-se com este caso que mesmo lesões aparentemente simples que não comprometem a locomoção podem levar a complicações e maior tempo de internamento devido à alteração do controle da micção.

Cinco dos 29 animais que foram submetidos a tratamento conservativo receberam o neuroprotetor SSMP. Dois animais receberam a primeira dose de $30 \mathrm{mg} / \mathrm{kg}$ e a segunda dose de $15 \mathrm{mg} / \mathrm{kg}$, e três animais receberam dose única de $30 \mathrm{mg} / \mathrm{kg}$. Os animais que receberam duas doses apresentavam lesão TL grau 4 e se recuperaram totalmente. Os dois pacientes apresentaram efeitos colaterais, como diarreia e vômito. Entre os três animais que receberam uma dose de SSMP, um com lesão TL grau 3 se recuperou totalmente, um com lesão TL grau 5 não apresentou recuperação e um com lesão medular lombar grau 5 morreu duas semanas após 
o trauma por causa desconhecida,. Vinte e quatro dos 29 animais não receberam SSMP, sendo que 18 deles recuperaram-se, três não apresentaram recuperação e três morreram. Pointillart et al. (2000) e Suberviola et al. (2008) demonstraram a ausência de benefício do tratamento com SSMP em pacientes humanos com lesão medular, relatando ainda um risco aumentado de complicações infecciosas e metabólicas, como a hiperglicemia. No presente estudo não houve diferença na recuperação dos pacientes que receberam o SSMP em relação aos que não receberam, entretanto o número de animais que recebeu o SMMP foi pequeno, quando comparado aos que não receberam. Acrescenta-se ainda que três pacientes apresentaram efeitos colaterais citados em literatura, como vômito e diarreia (Araújo et al. 2009, Webb et al. 2010). Segundo Kube \& Olby (2008), 33 a $90 \%$ dos casos que recebem SSMP apresentam complicações sérias como diarreia, melena, êmese, hematoquezia, anorexia, e segundo o Consenso Humano sobre o tratamento do trauma medular da Associação Americana de Neurocirurgiões, o SSMP não é mais considerado como padrão ou diretriz, e sim uma opção da qual deve-se ter consciência que os efeitos colaterais podem ser maiores que os benefícios (Bush al. 2007, Kube \& Olby 2008, Rabinowitz et al. 2008). Apesar do número pequeno de animais que receberam SSMP, observou-se que a administração deste fármaco não beneficiou os pacientes, além de ter causado efeitos colaterais e assim os autores do presente trabalho não recomendam o uso de SSMP no tratamento do trauma medular.

Treze animais apresentaram postura de Schiff-Sherrington. Oito dos 13 animais foram submetidos à eutanásia devido à não recuperação neurológica, dois dos 13 animais apresentaram recuperação funcional total, em um dos 13 animais houve recuperação parcial, e dois moreram (Fig.4). Esta postura é uma síndrome clínica vista em casos severos de trauma à medula espinhal no segmento toracolombar (Bali et al. 2009). Os três animais que apresentaram recuperação funcional total ou parcial possuíam a sensibilidade profunda intacta, o que está de acordo com Bagley et al. (1999), Bergman et al. (2000a) e De Lahunta \& Glass (2009), que afirmam que a presença ou ausência da percepção da dor profunda é o indicador mais importante para o prognóstico do que a presença da postura de Schiff-Sherrington.

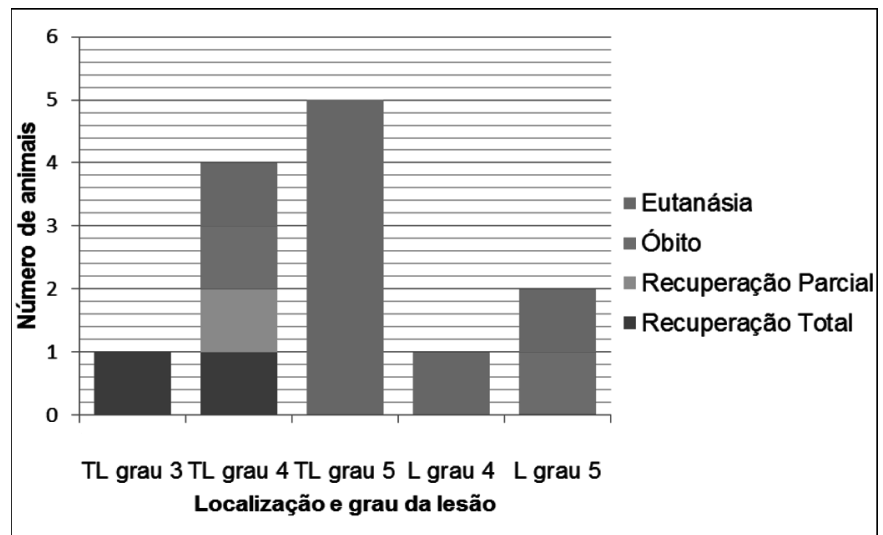

Fig.4. Recuperação funcional em treze animais com traumatismo espinomedular que apresentaram postura de Schiff-Sherrington (Londrina, 2011).
O objetivo do tratamento do trauma medular é fornecer um meio no qual o tecido neural possa recuperar sua função ótima, e seus objetivos são limitar as lesões adicionais causados pela instabilidade e aliviar a compressão restabelecendo a orientação anatômica das vértebras (Jeffery 2010). De acordo com estes princípios, o tratamento cirúrgico seria o ideal (Sharp \& Wheeler 2005, Jeffery 2010). Entretanto, mesmo na medicina humana ainda existem controvérsias sobre o melhor momento para a realização da cirurgia (Kube \& Olby 2008), principalmente devido à coexistência de traumas a vários órgãos e risco anestésico que pode piorar a perfusão medular (Jeffery 2010). No presente estudo, vários fatores levaram à opção pelo tratamento conservativo. Segundo Kube \& Olby (2008), se a cirurgia não puder ser realizada devido a restrições financeiras ou condição clínica do paciente, o tratamento médico pode ser uma opção, conforme observado no presente estudo e relatado por vários autores (Selcer et al. 1991, Hawthorne et al. 1999, Grasmueck \& Steffen 2004, Sharp \& Wheeler 2005). Nas lesões cervicais o resultado foi bom com tratamento conservativo, mesmo nos casos em que foi observada tetraparesia ou tetraplegia. Nas lesões toracolombares, a sensibilidade profunda intacta foi o principal determinante para uma boa evolução do quadro.

Ainda existem muitas dúvidas na escolha da melhor conduta no manejo do trauma medular e devido ao fato de seres humanos serem bastante afetados por este tipo de lesão a procura por um tratamento ideal é incessante. $\mathrm{Na}$ prática clínica muitas variáveis influenciam na decisão do veterinário, e estudos com maior número de animais submetidos ao tratamento cirúrgico são necessários para confrontar estes resultados.

\section{CONCLUSÕES}

Com base nos resultados pode-se concluir que a principal causa de lesão medular em cães e gatos foi o atropelamento, e embora mais da metade dos animais tenha sido trazido um dia após o trauma, o tempo entre o trauma e o atendimento não influenciou na recuperação dos pacientes.

Os segmentos medulares torácicos e lombares foram mais acometidos que os cervicais.

0 número de animais sobreviventes foi praticamente igual ao de não sobreviventes, e os fatores que influenciaram na mortalidade ou opção pela eutanásia foram a localização da lesão no segmento toracolombar e a perda da sensibilidade dolorosa profunda.

0 tratamento conservativo foi uma opção viável, permitindo a recuperação de 1/3 dos animais atendidos.

Agradecimentos.- À Universidade Estadual de Londrina/PROPPG, pela concessão de bolsa PIBIC para realização deste trabalho. À Prof ${ }^{a}$ Dr $^{a}$ Roberta Lemos Freire do Departamento de Medicina Veterinária Preventiva/ UEL, pela realização da análise estatística deste trabalho. Aos residentes do HV/UEL, pela disposição no atendimento e preenchimento das fichas dos animais.

\section{REFERÊNCIAS}

Araújo B.M., Bahr Arias M.V. \& Tudury E.A. 2009. Paraplegia aguda com perda da percepção de dor profunda em cães: revisão de literatura. Clínica Vet. 81:70-82. 
Arias M.V.B., Severo M.S. \& Tudury E.A. 2007. Trauma medular em cães e gatos: revisão da fisiopatologia e do tratamento médico. Semina, Ciênc. Agrárias 28:115-134.

Arias M.V.B., Nishioka C.M., Garcia C.O., Reia A.Z., Júnior D.B. \& Marcasso R.A. 2007. Avaliação dos resultados clínicos após cirurgia descompressiva em cães com doença do disco intervertebral. Arq. Bras. Med. Vet. 59:1445-1450.

Bagley R.S., Harrington M.L., Silver G.M., Cambridge A.J., Connors R.L. \& Moore M.P. 1999. Exogenous spinal trauma: clinical assessment and initial management. Compend. Contin. Educ. Vet. 21:1138-1144.

Bali M.S., Lang J., Jaggy A., Spreng D., Doherr M.G., Forterre F. 2009. Comparative study of vertebral fractures and luxations in dogs and cats. Vet. Comp. Orthop. Traumatol. 22:47-53.

Bentubo H.D.L., Tomaz M.A., Bondan E.F. \& Lallo M.A. 2007. Expectativa de vida e causas de morte em cães na área metropolitana de São Paulo (Brasil). Ciência Rural 37:1021-1026.

Bergman R. 2000. Spinal cord injury. Vet. Med. 95:845.

Bergman R., Lanz O. \& Shell L. 2000a. Acute spinal cord trauma: mechanisms and clinical syndromes. Vet. Med. 95:846-849.

Bergman R., Lanz O. \& Shell L. 2000b. Initial assessment of patients with spinal cord trauma. Vet. Med. 95:851-53.

Bergman R., Lanz 0. \& Shell L. 2000c. A review of experimental and clinical treatments for acute spinal cord injury. Vet. Med. 95:855-866.

Bruce C.W., Brisson B.A. \& Gyselinck K. 2008. Spinal fracture and luxation in dogs and cats: a retrospective evaluation of 95 cases. Vet. Comp. Orthop. Traumatol. 3:280-284.

Bush W.W., Tiches D.M., Kamprad C., Murtaugh R.J. \& Barr C.S. 2007. Hemilaminectomy without methylprednisolone sodium succinate for acute thoracolumbar disk disease in 51 non-ambulatory dogs. J. Vet. Emerg. Crit. Care. 17:72-76.

Campos M.F., Ribeiro A.T., Listik S., Pereira C.A.B. \& Sobrinho J.A. 2008. Epidemiologia do traumatismo da coluna vertebral. Revta Col. Bras. Cirur. 35:88-93.

Chai O., Johnston D.E. \& Shamir M.H. 2008. Bite wounds involving the spine: characteristics, therapy and outcome in sevem cases. Vet J. 175(2):259-265.

Defino H.L.A. 1999. Trauma raquimedular. Simpósio: Trauma II. Medicina, Ribeirão Preto, 32:388-400.

De Lahunta A. \& Glass E. 2009. Small animal spinal cord disease, p.243284. In: De Lahunta A. \& Glass E. (Eds), Veterinary Neuroanatomy and Clinical Neurology. W.B. Saunders, St Louis.

Fighera R.A., Silva M.C., Souza T.M., Brum J.S., Kommers D., Graça D.L., Irigoyen L.F. \& Barros C.S.L. 2008a. Aspectos patológicos de 155 casos fatais de cães atropelados por veículos automotivos. Ciênc. Rural 38(5):1375-1380.

Fighera R.A., Souza T.M., Silva M.C., Brum J.S., Graça D.L., Kommers D., Irigoyen L.F. \& Barros C.S.L. 2008b. Causas de morte e razões para eutanásia de cães da Mesorregião do Centro Ocidental Rio-Grandense (19652004). Pesq. Vet. Bras. 28:223-230.

Fontelles M.J.P. \& Mantovani M. 2000. Trauma torácico: fatores de risco de complicações pleuropulmonares pós-drenagem pleural fechada. Revista Col. Bras. Cirur. 27(6):400-407.

Grasmueck S. \& Steffen F. 2004. Survival rates and outcomes in cats with thoracic and lumbar spinal cord injuries due to external trauma. J. Small Anim. Pract. 45:284-288.

Hawthorne J.C., Blevins W.E., Wallace L.J., Glickman N. \& Waters D.J. 1999. Cervical vertebral fractures in 56 dogs: a retrospective study. J. Am. Anim. Hosp. Assoc. 35:135-146.
Jeffery N.D. \& Blakemore W.F. 1999. Spinal cord injury in small animals. Vet Rec. 145:183-190.

Jeffery N.D. 2010. Vertebral fracture and luxation in small animals. Vet. Clin. North Am., Small. Anim. Pract. 40:809-828.

Jorge S.M.C. 2009. Traumatismos medulares em canídeos. Dissertação de Mestrado Integrado em Medicina Veterinária, Faculdade de Medicina Veterinária, Universidade Técnica de Lisboa, Lisboa, Portugal. 85p.

Kube S.A. \& Olby N.J. 2008. Managing acute spinal cord injuries. Compend. Contin. Educ. Vet. 30:496-504

Olby N. 2010. The pathogenesis and treatment of acute spinal cord injuries in dogs. Vet. Clin. North Am. Small. Anim. Pract. 40:791-807.

Olby N., Levine J., Harris T., Muñana K., Skeen T. \& Sharp N. 2003. Long-term functional outcome of dogs with severe injuries of the thoracolumbar spinal cord: 87 cases (1996-2001). J. Am. Vet. Med. Assoc. 222:762-769.

Platt S.R. \& Olby N.J. 2010. Neurological emergencies, p.320-336. In: Ibid. (Eds), Manual of Canine and Feline Neurology. British Small Animal Veterinary Association, Gloucester, England.

Pointillart V., Petitjean M.E., Wiart L., Vital J.M., Lassié P., Thicoipé M. \& Dabadie P. 2000. Pharmacological therapy of spinal cord injury during the acute phase. Spinal Cord 38:71-76.

Rabinowitz R.S., Eck J.C., Harper C.M.J., Larson D.R., Jimenez M.A., Parisi J.E., Friedman J.A., Yaszemski M.J. \& Currier B.L. 2008. Urgent surgical decompression compared methylprednisolone for the treatment of acute spinal cord injury: a randomized prospective study in beagle dogs. Spine 1:2260-2268.

Santos R.P., Mazzanti A., Beckmann D.V., Berté L., Ripplinger A., Neto D.P. \& Baumhardt R. 2011. Recuperação funcional em cães com doença do disco intervertebral toracolombar sem percepção à dor profunda: 37 casos (2002-2010). Pesq. Vet. Bras. 31:345-349.

Selcer R.R., Bubb W.J. \& Walker T.L. 1991. Management of vertebral column fractures in dogs and cats: 211 cases (1977-1985). J. Am. Vet. Med. Assoc. 11:1965-1968.

Sharp N.J.H. \& Wheeler S.J. 2005. Trauma, p.281-318. In: Ibid. (Eds), Small Animal Spinal Disorders: diagnosis and surgery. Elsevier Mosby, Philadelphia.

Souza Junior M.F., Neves A.C.A., Medeiros A.A.A. \& Jallageas D.N. 2003. Características epidemiológicas do trauma raquimedular na Amazônia: análise prospectiva de 250 casos. J. Bras. Neurocir. 14:97-104.

Spackman C.J., Caywood D.D., Feeney D.A. \& Johnston G.R. 1984. Thoracic wall and pulmonary trauma in dogs sustaining fractures as a result of motor vehicle accidents. J. Am. Vet. Med. Assoc. 185(9):975-977.

Suberviola B., Gonzalez-Castro A., Llorca J., Ortiz-Melón F. \& Miñambres E. 2008. Early complications of high-dose methylprednisolone in acute spinal cord injury patients. Injury 39:748-752.

Tudury E.A., Júnior D.B., Chioratto R., Silva S.R.A.M., Roehsig C., Rocha L.B., Almeida A.C.M., Kemper B. \& Maciel M.J.S. 2006. Denervação dos esfíncteres uretrais, via celiotomia pré-púbica, em três gatos paraplégicos. Clínica Vet. 60:54-58.

Trunkey D.D. 1983. Trauma. Scientific American 249(2):28-35.

Webb A.A., Ngan S. \& Fowler D. 2010. Spinal cord injury. II. Prognostic indicators, standards of care, and clinical trials. Can. Vet. J. 51:598-604.

Weh J.M. \& Kraus K.H. 2007. Use of a four pin and methylmethacrylate fixation in L7 and the iliac body to stabilize lumbosacral fracture-luxations: A clinical and anatomic study. Vet. Surg. 36:775-782.

Zotti A., Gianesella M., Gasparinetti N., Zanetti E. \& Cozzi B. 2011. A preliminary investigation of the relationship between the "moment of resistance" of the canine spine, and the frequency of traumatic vertebral lesions at different spinal levels. Res. Vet. Sci. 90:179-184. 